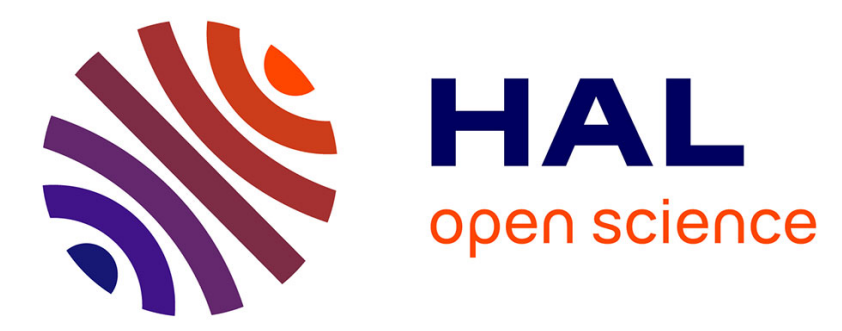

\title{
Psychophysical Power Optimization of Friction Modulation for Tactile Interfaces
}

Thomas Sednaoui, Eric Vezzoli, David Gueorguiev, Cedrick Chappaz, Betty Lemaire-Semail

\section{- To cite this version:}

Thomas Sednaoui, Eric Vezzoli, David Gueorguiev, Cedrick Chappaz, Betty Lemaire-Semail. Psychophysical Power Optimization of Friction Modulation for Tactile Interfaces. Haptics: Perception, Devices, Control, and Applications, Jul 2016, London, United Kingdom. 10.1007/978-3-319-423241_35. hal-01342010

\section{HAL Id: hal-01342010 \\ https://inria.hal.science/hal-01342010}

Submitted on 11 Jul 2016

HAL is a multi-disciplinary open access archive for the deposit and dissemination of scientific research documents, whether they are published or not. The documents may come from teaching and research institutions in France or abroad, or from public or private research centers.
L'archive ouverte pluridisciplinaire HAL, est destinée au dépôt et à la diffusion de documents scientifiques de niveau recherche, publiés ou non, émanant des établissements d'enseignement et de recherche français ou étrangers, des laboratoires publics ou privés. 


\title{
Psychophysical Power Optimization of Friction Modulation Tactile Interfaces
}

\author{
Thomas Sednaoui ${ }^{1,2}$, Eric Vezzoli ${ }^{1}$, David Gueorguiev ${ }^{3}$, Cedrick Chappaz ${ }^{3}$ and Betty Lemaire- \\ Semail $^{1}$ \\ ${ }^{1}$ Univ. Lille, Centrale Lille, Arts et Metiers ParisTech, HEI, EA 2697 - L2EP - Laboratoire d'Electro- \\ technique et d'Electronique de Puissance, F-59000 Lille, France \\ eric.vezzoli@ed.univ-lillel.fr; betty.semail@polytech-lille.fr \\ ${ }^{2}$ STMicroelectronics, Crolles F38920, France \\ thomas.sednaouilst.com \\ ${ }^{3}$ Institute of Neuroscience (IoNS), Université Catholique de Louvain, Brussels, Belgium 1200 \\ david.gueorguiev@uclouvain. be \\ ${ }^{3}$ HAP2U, Grenoble 38000, France \\ cedrick.chappaz@hap2u.net
}

\begin{abstract}
Ultrasonic vibration and electrovibration can modulate the friction between a surface and a sliding finger. The power consumption of these devices is critical to their integration in modern mobile devices such as smartphones. This paper presents a simple control solution to reduce up to $68.8 \%$ this power consumption by taking advantage of the human perception limits.
\end{abstract}

Keywords: ultrasonic lubrication, electrovibrations, tactile device, friction control, power consumption

\section{Introduction}

The last few years have seen the emergence of ubiquitous mobile devices and tactile interfaces. The abundance of these novel interfaces raised the interest in touch based human-machine interactions and highlighted the lack of natural touch feedback in the existing generation of tactile displays. The problem was partly responsible for the slow adoption of the technology among consumers. Currently, multiple solutions are being explored to deliver improved haptic feedback on existing mobile platforms such as smartphones or tablets; one such feedback technology, vibrotactile stimulation, is already incorporated on most platforms but only provides a general vibration sensation to the hand and finger of users [1]. To improve upon this, tactile based solutions have been proposed in recent years such as electrovibration [2] and Ultrasonic Lubrication (UL) [3] [4] [5]. These technologies have very different means of action but ultimately affect the dynamic friction between the finger and the tactile plate in a similar manner [6]. Integration into mobile devices creates multiple challenges in both technologies. The power consumption is one of these challenges considering the already limited endurance of mobile devices. 
Both UL and electrovibration technologies use their friction control capabilities to create a reproduction of simple textures. This reproduction is typically applied by alternating quickly states of high and low friction while the user moves its finger across the active surface [2]. In most cases, this switching is simply implemented by applying a square modulation signal to the friction control device [7]. Perception of a specific texture is driven by the spatial frequency pattern of this texture. This pattern can then be recreated in the time domain by modulating the active phases in function of the exploration speed. This study explores the possibility of reducing the duty cycle of such modulation while maintaining the same texture perception. Reducing the duty cycle of modulation affects the total active time and thus the final power consumption of the tactile device.

We chose an UL device to implement this study. This choice was made from due to two main factors. First an UL device presents higher power consumption in active state than electrovibration devices for a similar form factor. This is due mostly to continuous damping of the air against the vibrating plate [8]. A second factor is that electrovibration presents a perception degradation when repeated sliding of the finger are performed. This is caused by the deposition of sweat that shields the finger from the electrical field [9].

The current paper demonstrates that power consumption in a friction modulation device can be reduced significantly by reducing the duty cycle of the modulation stimulation. A power reduction scheme is proposed and validated using psychophysics measures on five participants performing a discrimination task on an UL device. A wide range of modulation frequencies is tested to validate the proposed scheme across the frequency perception range of the human fingertip.

\section{Experimental Setup}

To perform this study, we developed a specific stimulator designed to deliver controlled and repeatable stimulations. First, the general experimental system is described and then the UL display that was specifically developed.

\subsection{Global System Description}

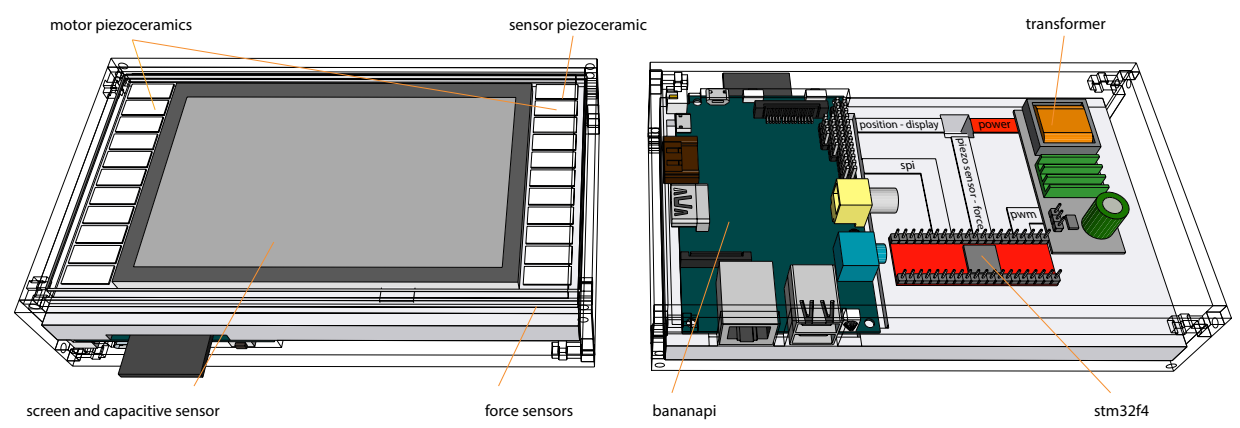

Fig. 1. Device architecture [10] 
In order to control the modulation frequency and the vibration amplitude of the haptic plate, a new device was specifically developed. As seen in Fig. 1 this system includes both visual and haptic feedback. Finger touch position is directly acquired using a capacitive touch screen. Computation and control of the experiment is separated in two parts: a "High level" signal using the banana pi (Shenzhen LeMaker Technology Co. Ltd, China) single board computer featuring a $1 \mathrm{GHz}$ ARM Cortex-A7 dual-core CPU with $1 \mathrm{~GB}$ of ram. A "Low Level" signal generation is implemented in a separate DSP microcontroller (stm32f4, STMicroelectronics, France) running at $164 \mathrm{MHz}$. In this setup "High level" computing refers to the display of the instruction to the user, selection of the haptic signal commands and storage of the results. The signal generation microcontroller for its part applies commands from the board computer to create the necessary waveforms for the friction modulation. The communication between the microcontroller and the single board pc is provided by an SPI bus working at $10 \mathrm{kHz}$. In order to insure the fastest amplitude rise time in this study, an external amplifier is used to drive the piezoceramic motors as in [3].

The single board computer is connected to a 5 inches flat capacitive touch screen (BananaLCD-5"-TS, Marel, China) providing the finger position input and display output, where the sampling frequency of the finger position is $62 \mathrm{~Hz}$. This LCD display gives visual confirmation of the experiment goals during the measures. A second visual system using a computer screen is used to display comfortably the controls of the experiments to each participant.

\subsection{Ultrasonic Vibrating Plate}

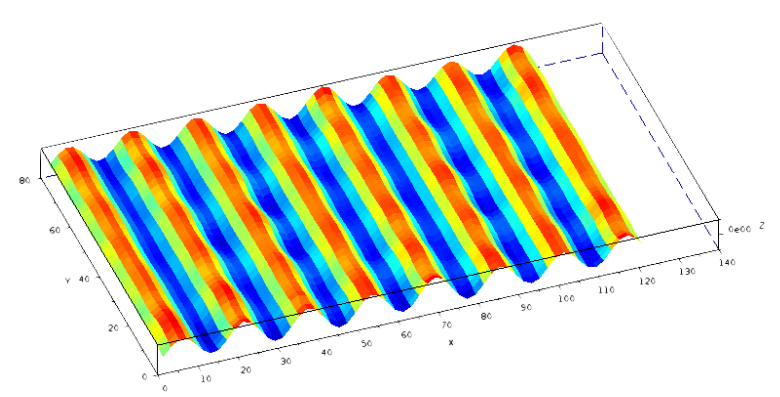

Fig. 2. Cartography of the ultrasonic vibrating plate

The ultrasonic vibrating plate implemented in the device is specifically designed to provide the best modulation bandwidth [10]. The glass plate measures $154 \times 81 \times 1.6 \mathrm{~mm}$ resonating at 60750 $\mathrm{Hz}$, where the half length of the vibration mode is $8 \mathrm{~mm}$. 22 piezoceramics, $14 \times 6 \times 0.5 \mathrm{~mm}$, are mounted at the side of the plate along the extremum of deformation, 20 used as motors and 2 as vibration sensors. Their unglued electrode was cut along the nodal and each side connected to the amplifier to create a virtual mass on the glued face of the ceramic. The cartography of the vibration amplitude of the plate is reported in Fig. 2.

Power consumption associated with reduction of the duty cycle has been measured with the help of a Tectronics oscilloscope and a current probe. A linear relationship was found between the consumed power and a duty cycle ranging from $5 \%$ to $95 \%$. 


\section{Experimental protocols}

This section presents the psychophysical protocols used in the two following experiments. A first experiment was conducted to determine the capacity of participants to distinguish differences in the duration of a single tactile stimulation. In a second time an experimental protocol to measure the achievable duty cycle reduction for a range of modulation frequencies is presented.

In all experiments, the participants were interacting with the tactile device described in Chapter 2 . Five subjects aged between 25 and 30 participated to this study ( $4 \mathrm{~m}$. and $1 \mathrm{f}$.). The UL tactile interface sometimes makes a slight noise when alternating active and passive states. Therefore, to prevent any influence of this noise on the participant's perception, the subjects had to wear noise reduction headphones and a white noise was also diffused in the room during the experiments. For each trial the participants had to move their finger from left to right on the screen which generates a stimulation but were free to choose the force and speed of exploration. The participants had to lift their fingers from the plate after each sliding on the plate.

\subsection{Minimum signal duration}

The minimal signal duration discrimination experiment aim was to determine the capacity to perceive differences in the length of the friction reduction. From this psychometric function it was then possible to extract the just noticeable difference (JND) of signal duration for humans interacting with the UL tactile device. The experiment was based on a forced choice task with a constant pool of stimuli (constant stimuli method). The participants had to compare the length of two stimuli, displayed in sequential order. A reference value of signal duration was always randomly set as one of the two stimuli presented in the discrimination task. The other stimulus was a test value to compare against the baseline. The participants explored the two stimuli up to 3 times and was then asked to report which stimulus offered the "shortest" stimulation. They had to choose one of the signals even if not sure of the answer.

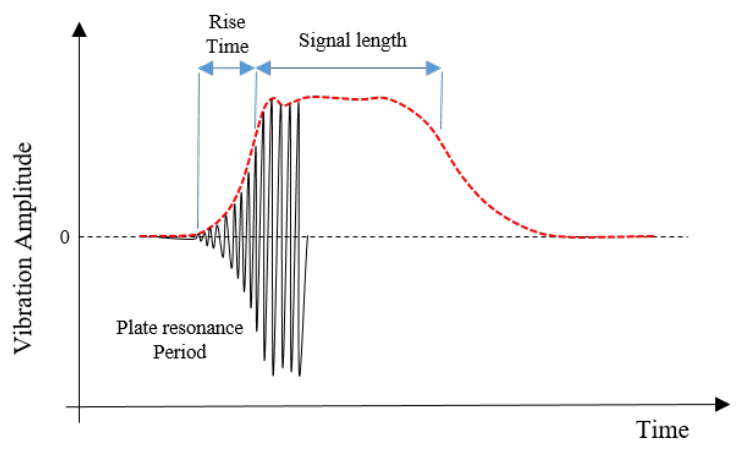

Fig. 3. Voltage amplitude of the signal duration experiment

Test signals were presented in a pseudorandom order where one of the signals was always the baseline and the other selected from a list of five signal length calibrated in advance. The 
possible time durations were $1.6,2.4,3.2$ to $4 \mathrm{~ms}$ to be compared with a baseline of $0.8 \mathrm{~ms}$. The experiment was stopped after a signal of each length had been presented 10 times against the reference. Each of the five subjects was thus presented to a total of 40 signal comparisons.

\subsection{Duty Cycle modulation}

The aim of the second experiment was to determine the maximum duty cycle reduction achievable while keeping the same perception of the modulation. The potential duty cycle reduction was assessed for multiples frequencies within the human perception range. To this end, this experiment was repeated on a range of modulation frequency for each participant.

For each set of modulation frequencies, the psychometric function and the JND of the duty cycle discrimination was determined. Similarly, to the previous experiment a constant stimuli method was used. The participants explored the two stimuli up to 3 times and were then asked to report which stimulus offered the "strongest" stimulation. They had to choose one of the signals even if not sure of the answer.

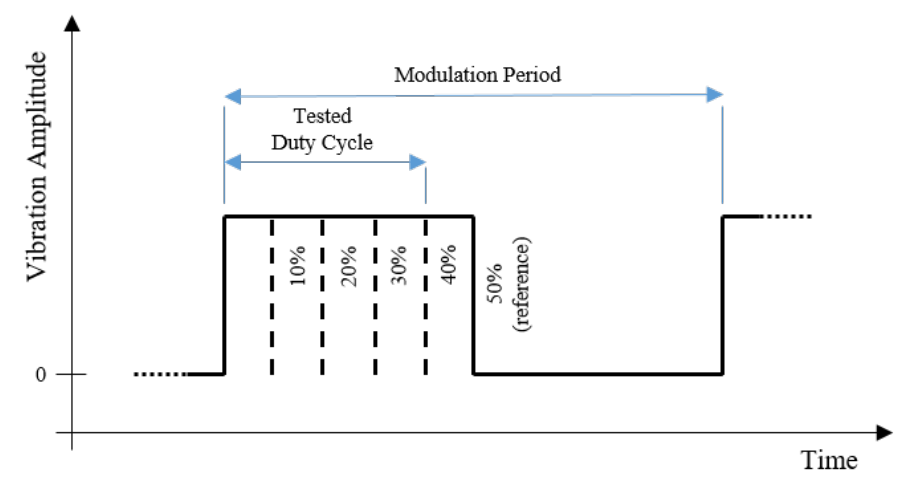

Fig. 4. Reduction cases of the Duty Cycle

As in the first experiment, the test signals were presented in a pseudorandom order where one of the signals was always the reference and the other selected from a list of 4 duty cycles calibrated in advance. The list of possible duty cycles was $10 \%, 20 \%, 30 \%$, and $40 \%$, with the square $50 \%$ duty cycle used as the reference stimulus (Fig. 4). The experiment was once again stopped after each duty cycle was presented 10 times against the reference.

The same protocol was implemented 4 times for different modulation frequencies: $10 \mathrm{~Hz}$, $20 \mathrm{~Hz}, 40 \mathrm{~Hz}$ and $80 \mathrm{~Hz}$. To prevent fatigue, the 4 experiments were divided in 2 sessions separated by a one-hour break. Each of the five subjects was thus presented to a total of 160 signal comparison tests to extract the 4 duty cycle psychometric functions and JND values.

\subsection{Psychophysical analysis}

In each task, the psychophysical threshold was evaluated by fitting a logistic psychometric function based on the method of maximum likelihood to the psychophysical performance of the 
participants. The fitting was implemented by using the version 1.81 of the Palamedes toolbox [11] (Kingdom \& Prins).

\section{$4 \quad$ Results}

\subsection{Minimum Signal Length}

Results from the five subjects are presented. All the answers from the five participants were averaged together and the standard deviation calculated. The data obtained during the trial are shown in Fig. 5.

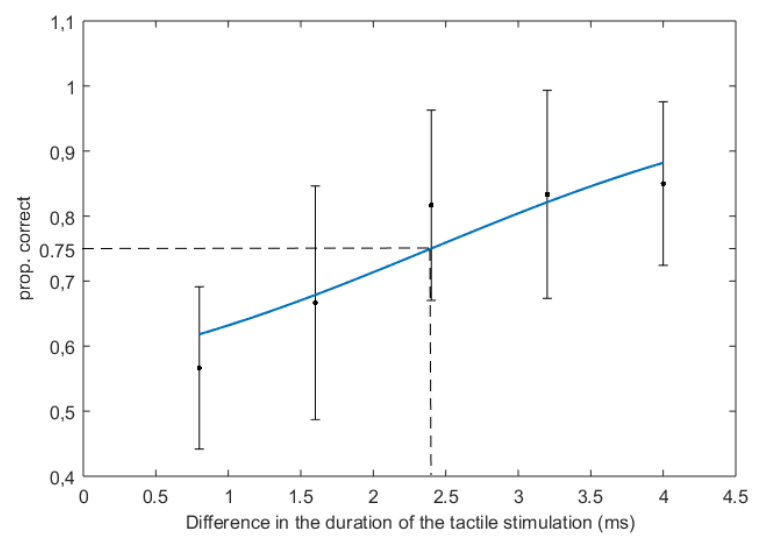

Fig. 5. Just noticeable difference of signal length. Error bars represent the standard deviation and blue line is a fit of a logistic psychometric function to the result plot.

To compute the just noticeable difference (JND), which represents the minimum signal length difference that can be reliably discriminated, the percent of the time that the participants responded correctly as a function of the difference in the signal length was calculated. A logistic psychometric function bounded between 0.5 and 1 was fitted to the resulting plot. A value of 0.5 on the $\mathrm{Y}$ axis represents a situation where the participant performs at chance level. On the contrary a value of 1 represents $100 \%$ correct discrimination from the participant. 
For a forced choice task with two alternatives, the JND is typically set as $75 \%$ of correct answers. We computed it by interception of the psychometric function at $Y=0.75$ and the overall JND for the five participants was therefore estimated at $2.45 \mathrm{~ms}$.

\subsection{Duty Cycle Modulation}

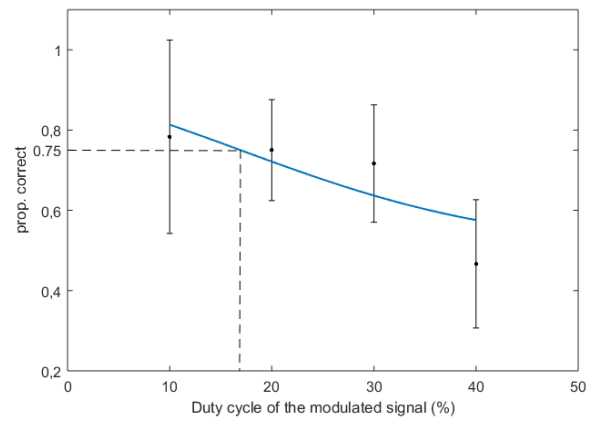

Fig. 6. A JND of duty cycle at $10 \mathrm{~Hz}$ modulation

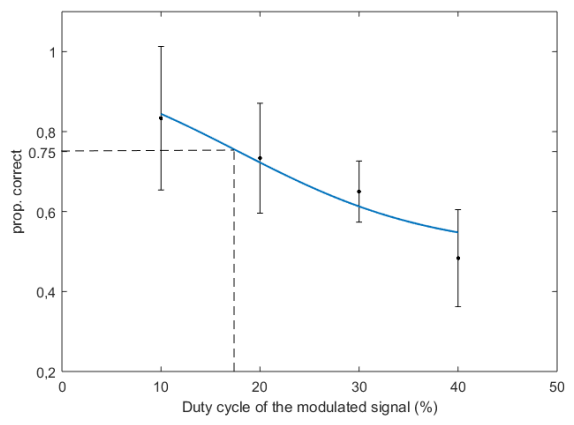

B JND of duty cycle at $80 \mathrm{~Hz}$ modulation

In this experiment, four modulation frequencies were explored in separate blocks. Similarly to the time length experiment, the JND, which represented minimum duty cycle that can be reliably discriminated from a reference duty cycle of $50 \%$, was computed by estimating the interception point of a psychometric function fitted to the answers of the participants and a proportion of correct answers of 0.75.The overall JND's for the five subjects who participated in this experiment were calculated at a duty cycle of respectively $16.5,17.5,11$ and $17.5 \%$ for modulation frequency of 10, 20, 40 and $80 \mathrm{~Hz}$.

\section{Discussions}

This section first discusses the impacts of the experiment's results on UL power consumption. A second part describes the improvements and future work to complete and validate the proposed control scheme.

\subsection{Impacts on the energy consumption}

The results presented in chapter 4.2 show that reducing the duty cycle of a friction modulation signal is not always perceivable by the participant. Moreover, these JND values represent a reduction relative to a standard duty cycle of $50 \%$ of respectively $67,65,78$ and $65 \%$ for modulation frequency of 10, 20, 40 and $80 \mathrm{~Hz}$.

As described in chapter 2.2, the UL device power consumption is reduced linearly with the application of smaller duty cycles. Fig. 7 shows a stable reduction of the duty cycle across the studied frequency spectrum. The modulation frequency does not seem to impact the duty cycle JND. 


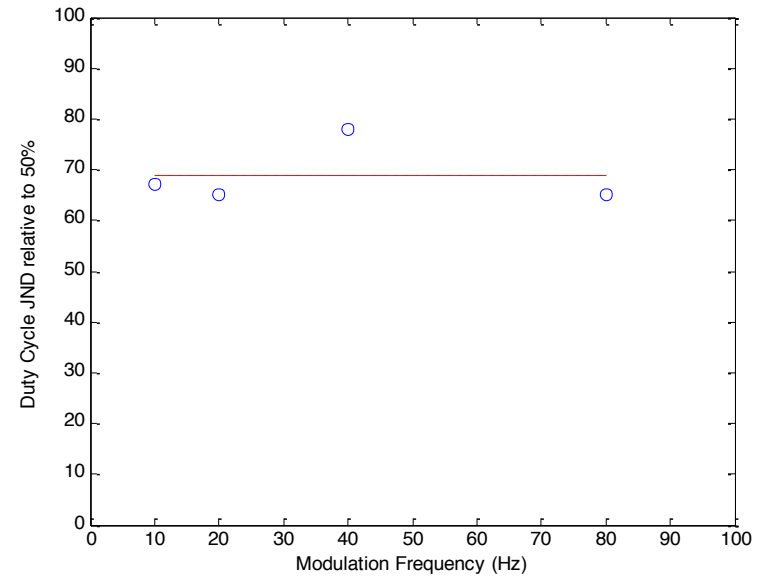

Fig. 7. JND of the duty cycle in function of the modulation frequency. JND is relative to a standard duty cycle of $50 \%$. Dotted line represents the mean JND across the spectrum.

An average of $68.8 \%$ power reduction is available across the 10 to $80 \mathrm{~Hz}$ modulation frequency without perceivable difference to the participant. It is to be noted that this reduction of power consumption should be also similar for electrostatic devices since the usual approach is to apply an amplitude modulated sinusoidal voltage in active state.

The indistinguishability of signals under $2.45 \mathrm{~ms}$ allow us to reduce a single pulse stimulation to the shortest possible without any difference to the tactile users. This limit could be used to reduce the power consumption in specific usage cases.

\subsection{Prospective}

The JND of $17.5 \%$ found for $80 \mathrm{kHz}$ represent burst of $2.13 \mathrm{~ms}$ witch is consistent with the indistinguishability of signals under $2.45 \mathrm{~ms}$ shown in chapter 4.1 . This limit of indistinguishability could mean a modification of the relationship shown in Fig. 7 for higher modulation frequencies. The range of modulation frequency $(10$ to $80 \mathrm{~Hz})$ in the current paper is limited by the time response of the haptic stimulator. To solve this issue a second haptic stimulator with a smaller time response will be designed to reach a modulation frequency closer to the $500 \mathrm{~Hz}$ of human perception. This device will allow us to estimate the limits of human perception of the friction modulation and ultimately relate it to perceptual bandwidth of the fingertip mechanoreceptors.

The results presented in this paper are currently limited to 5 participants. These results will be validated in a subsequent study with a larger number of participants. 
Ultrasonic lubrication and electrovibration do not have the save power consumption while in active state. The current paper uses a device based on Ultrasonic lubrication as a proof of concept for the proposed texture simulation strategy. Further study will measure precisely the energy saving that can be expected for both technologies.

\section{Conclusion}

A general control scheme based on human perception to reduce power consumption in friction modulation devices is introduced. The results are validated using psychophysics measures on an ultrasonic lubrication device. A power reduction of up to $68.8 \%$ is confirmed for an ultrasonic device across the 10 to $80 \mathrm{~Hz}$ range of modulation studied.

\section{Acknowledgement}

This work was founded by the FP7 Marie Curie Initial Training Network PROTOTOUCH, grant agreement No. 317100.

This work has been supported by IRCICA USR 3380 Univ.Lille - CNRS (www.ircica.univlille1.fr)

\section{References}

[1] B. M. Schena, «Directional inertial tactile feedback using rotating masses». USA Brevet US7182691 B1, 27 February 2007.

[2] D. J. Meyer, . M. A. Peshkin et E. J. Colgate, «Fingertip friction modulation due to electrostatic attraction,» chez World Haptics Conference (WHC), 2013, 2013/4/14.

[3] M. Amberg, F. Giraud, B. Semail, P. Olivo, G. Casiez et N. Roussel, «STIMTAC: a tactile input device with programmable friction,» chez Proceedings of the 24th annual ACM symposium adjunct on User interface software and technology, 2011/10/16.

[4] F. Giraud, M. Amberg, B. Lemaire-Semail et G. Casiez, «Design of a transparent tactile stimulator,» Haptics Symposium (HAPTICS), pp. 485-489, 2012/3/5.

[5] T. Sednaoui, E. Vezzoli, B. Dzidek, B. Lemaire-Semail, C. Chappaz and M. Adams, "Experimental Evaluation of Friction Reduction in Ultrasonic Devices," in World Haptic, Chicago, 2015.

[6] E. Vezzoli, W. Ben Messaoud, M. Amberg, F. Giraud, B. Lemaire-Semail t M.-A. Bueno, «Physical and perceptual independence of ultrasonic vibration and electrovibration for friction modulation,» Haptics, IEEE Transactions on, vol. 8, n $\% 12$, pp. $235-239,2015$. 
[7] G. Frédéric, M. Amberg and B. Lemaire-Semail, «Merging two tactile stimulation principles: electrovibration and squeeze film effect,» in World Haptic (WHC), Daejeon, 2013.

[8] W. Ben Messaoud, E. Vezzoli, F. Giraud and B. Lemaire-Semail, «Pressure dependence of friction modulation in ultrasonic devices,» in World Haptic Conference, Versaille, 2015.

[9] K. A. Kaczmarek, K. Nammi, A. K. Agarwal, M. E. Tyler, S. J. Haase and D. Beebe, «Polarity Effect in Electrovibration for Tactile Display,» IEEE Transaction on Biomedical Engineering, vol. 53, n%110, pp. 2047-2054, 2006.

[10] E. Vezzoli, T. Sednaoui, M. Amberg, F. Giraud et B. Lemaire-Semail, «Texture Rendering Strategies with a High Fidelity - Capacitive Visual-Haptic Friction Control Device» Proposed in EuroHaptic, London, 2016.

[11] F. a. Kingdom et N. Prins, «Psychophysics: A Pratical Introduction,» 2009. 